\title{
The Importance of Accurate X-ray Energy Spectra for Modelling Dose Deposition with Monte Carlo Techniques
}

Introduction: There are many areas where accurate prediction of X-ray dose is important. A particularly relevant example is in clinical radiography, where patients are subjected to what is considered a known radiation dose to various sensitive organs. It is common practice to take X-ray spectra dictated by programs such as SpekCalc[1] or through simulation via Monte Carlo techniques such as GEANT4[2]. These spectra are then used for the simulation of patients in X-ray dose studies. This arises as it is often difficult and expensive to accurately characterise the spectrum of an X-ray source while modelling software is a simple and fast alternative. The aim of the study was to determine the reliability of such methods and if there is a potential for errors in dose prediction.

Methods: A Monte Carlo model was developed to predict the dose deposited in exposed materials for a given X-ray spectrum and these were compare to experimental measurements. To illustrate the importance of selecting the correct input spectrum for dose calculations, three different X-ray spectra were used in the modelling phase. The first was calculated via a commonly used analytical approximation (SpekCalc), the second resulted from a Monte Carlo model (based on the architecture and specification of the X-ray tube used in the experimental phase) and the third was from a direct measurement of the X-ray spectrum using a cadmiumtelluride spectrometer. The spectra are shown in Figure 1. It was expected that there should be good agreement between the modelled and measured spectrum however this was not the case. Also shown in Figure 1 is the Monte Carlo derived spectrum with additional 8 microns of tungsten filtration which matches the measured spectrum more accurately. The need for this additional filtration might be due to deposition of evaporated tungsten on the beam exit window or simply due to inaccuracies in the manufacturer provided datasheet.

To determine the dose (both in the model and experimentally), a simple setup was used which was comprised of layers of TLDs with different filter materials being placed between. The filters consisted of incremental thicknesses of copper ( 0.1 to $1 \mathrm{~mm}$ ), aluminium ( 1 to $10 \mathrm{~mm}$ ) and mixtures thereof. Once the model had been shown to yield accurate results it was adapted to exemplify the dose delivered to biological tissue. A simple chest model was developed, consisting of skin, bone and lung tissue. The dose delivered to each layer was computed for each input spectra, with each simulation using $10^{9}$ photons so that the dose errors were on the order of $\pm 0.01 \%$.

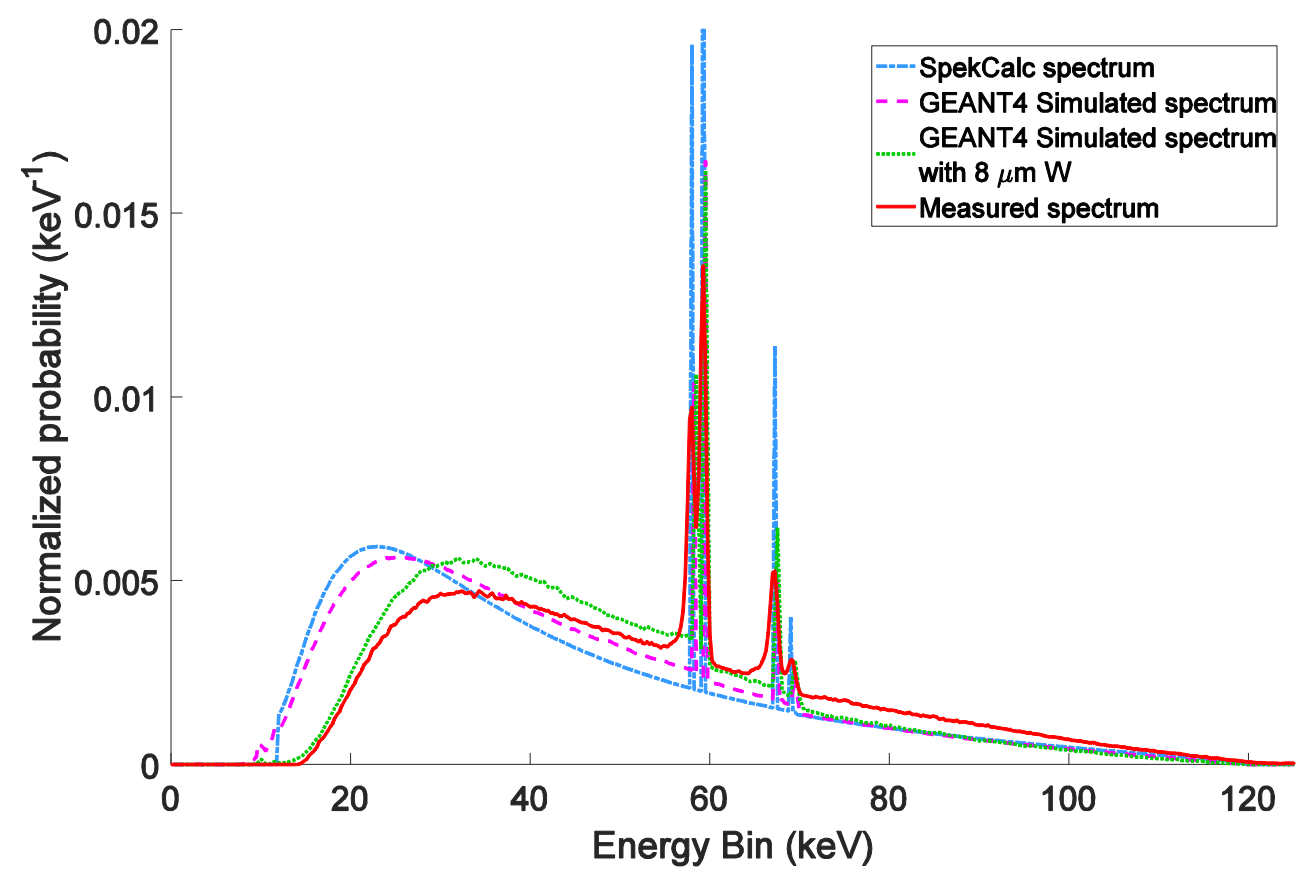

Figure 1: Four estimations for the spectrum of the same X-ray source (120 kVp, $1.2 \mathrm{~mA}$ ) using SpekCalc (blue), GEANT4 tube simulation (pink) and with extra tungsten filtration (green) and CdTe Spectrometrv (red). 


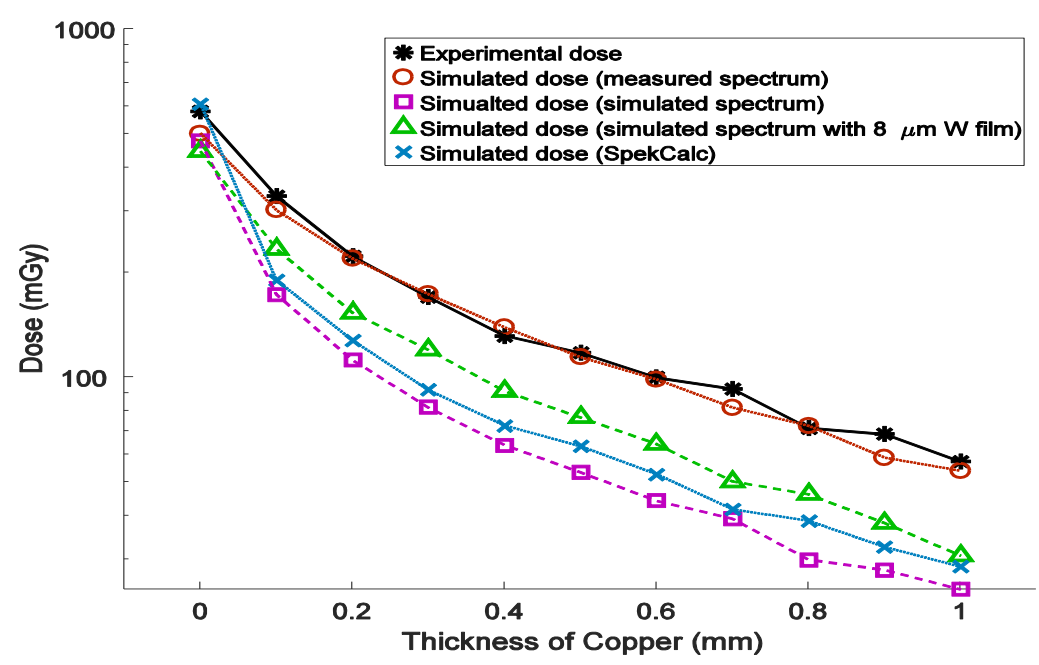

Figure 2: Dose deposited in TLDs (on a log scale) from experimental and simulated exposure to the $\mathrm{X}$-ray source through increasing layers of copper filtration.

Simulations used 4 input spectra.

Results and Discussion: The TLD exposure results are shown in Figure 2. It can be seen that as the filtration increases the different simulated spectra agree much less with reality, except when using the CdTe measured spectrum. This indicates that any dose predictions made using spectra that are slightly different from the real beam will become less and less accurate as the beam penetrates further through a patient.

To investigate this possibility, a basic simulation of a chest X-ray using the same spectra was run. The model consisted of cubic volumes composed of $2 \mathrm{~mm}$ of skin, $7 \mathrm{~mm}$ of bone and $120 \mathrm{~mm}$ of lung tissue and backed with two more layers of bone and skin. The results from this simulation are shown in table 1 . It can be seen that the spectra obtained from SpekCalc and the tube simulation gave an overestimation of the skin and bone dose by up to $20 \%$ and underestimated the lung tissue dose by up to $10 \%$. The disparity between these spectra increases with more filtration through the patient.

\begin{tabular}{|c|c|c|c|c|c|c|c|c|}
\hline \multirow{3}{*}{$\begin{array}{l}\begin{array}{c}\text { Dose (mGy) (\% } \\
\text { change) }\end{array} \\
\text { Tissue } \\
\text { Front Skin }\end{array}$} & \multicolumn{8}{|c|}{ Spectra } \\
\hline & \multicolumn{2}{|c|}{ CdTe Measured } & \multicolumn{2}{|c|}{ GEANT Sim } & \multicolumn{2}{|c|}{ SpekCalc } & \multicolumn{2}{|c|}{$\begin{array}{l}\text { GEANT Sim with } \\
8 \mu \mathrm{m} \text { W filter }\end{array}$} \\
\hline & 9.42 & - & 10.6 & $(+13 \%)$ & 11.3 & $(+20 \%)$ & 8.57 & $(-9.0 \%)$ \\
\hline Front Ribs & 34.6 & - & 37.0 & $(+6.9 \%)$ & 38.1 & $(+10 \%)$ & 32.0 & $(-7.4 \%)$ \\
\hline Lung Tissue & 2.39 & - & 2.14 & $(-11 \%)$ & 2.26 & $(-5.4 \%)$ & 1.92 & $(-20 \%)$ \\
\hline Back Ribs & 1.54 & - & 1.33 & $(-14 \%)$ & 1.42 & $(-8.1 \%)$ & 1.21 & $(-22 \%)$ \\
\hline Back Skin & 0.23 & - & 0.19 & $(-18 \%)$ & 0.21 & $(-10 \%)$ & 0.16 & $(-29 \%)$ \\
\hline
\end{tabular}

Table 1: A table showing the dose accumulated, in $\mathrm{mGy}$, by various anatomical volumes from a general chest $\mathrm{X}$-ray exposure and the percentage difference in dose these show from the measured spectrum

Conclusion: It has been shown that X-ray spectrum prediction software are not necessarily accurate. The resultant shifts in the output spectrum can lead to significant differences in radiation dose simulation and prediction. When these are used for the purposes of dose evaluation of patients, there is potential for a significant error in the calculation. These spectrum shifts could lead to inaccurate dose assessment for patients in medical treatment, resulting in an increased dose to internal sensitive tissue.

[1] G. Poludniowski, G. Landry, F. DeBlois, P. M. Evans, and F. Verhaegen, 'SpekCalc : a program to calculate photon spectra from tungsten anode x-ray tubes', Phys. Med. Biol., vol. 54, no. 19, p. N433, 2009.

[2] G. Barca et al., 'A powerful simulation tool for medical physics applications: Geant4', Nucl. Phys. B - Proc. Suppl., vol. 125, pp. 80-84, Sep. 2003. 